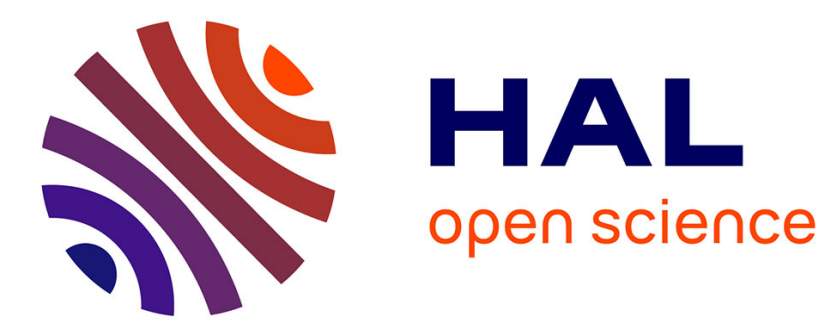

\title{
Periodic solutions versus practical switching control for sensorless Piecewise Affine systems (PWA)
}

Djekidel Kamri, Romain Bourdais

\section{To cite this version:}

Djekidel Kamri, Romain Bourdais. Periodic solutions versus practical switching control for sensorless Piecewise Affine systems (PWA). Journal of The Franklin Institute, 2017, 354 (2), pp. 917-937. 10.1016/j.jfranklin.2016.10.038 . hal-01513712

\section{HAL Id: hal-01513712 \\ https://hal.science/hal-01513712}

Submitted on 10 Jul 2017

HAL is a multi-disciplinary open access archive for the deposit and dissemination of scientific research documents, whether they are published or not. The documents may come from teaching and research institutions in France or abroad, or from public or private research centers.
L'archive ouverte pluridisciplinaire HAL, est destinée au dépôt et à la diffusion de documents scientifiques de niveau recherche, publiés ou non, émanant des établissements d'enseignement et de recherche français ou étrangers, des laboratoires publics ou privés. 


\title{
Periodic solutions versus practical switching control for sensorless Piecewise Affine systems (PWA)
}

\author{
Djekidel Kamri ${ }^{\mathrm{a}, *}$, Romain Bourdais ${ }^{\mathrm{b}}$ \\ ${ }^{a}$ Department of Electronic, Faculty of Technology, University Amar Thelidji, Laghouat, Algeria \\ ${ }^{\mathrm{b}}$ Automatic and Control Group, Supélec-IETR, Rennes Campus, France
}

\begin{abstract}
This work concerns the stabilization of general Piecewise Affine (PWA) systems without common equilibrium; the main objective consists in proposing a characterization of periodic solutions, by determining the critical parameters values of the cyclic behaviors. The proposed approach is based on the expansion of previous results on practical stabilization by switching. Due to the non-convex nature of general PWA synthesis problems, we primarily present a BMI formulation of the practical stabilization that is used to generate periodic solutions. More precisely, we characterize $\omega$-limit sets as periodic trajectories of the global PWA system in terms of special invariant sets of the practical stabilization method. This will avoid a posteriori subset inclusion checking, since the underlying set belongs to the admissible state space part. This approach generalizes previous results to obtain invariance conditions and the set $\omega$-limit points. A methodology and algorithms to compute periodic trajectories parameters are provided. Two illustrative examples are used for simulation, in particular the third order of Goodwin oscillator model is investigated as a non-uniform oscillatory complex system.
\end{abstract}

Keywords: Invariant Sets; LMI; Periodic Solutions; PWA Systems; Practical Switching Stabilization; $\omega$-Limits points

\section{Introduction}

The Piecewise Affine (PWA) systems modeling framework is the most appropriate representation of general hybrid systems that results from diverse engineering fields. PWA 
systems are characterized by a collection of different affine dynamics, each one is activated in a continuous state space region. These systems have received growing interest [1-6] in the last decades since they can approximate most nonlinear behaviors with high accuracy and they are advanced in practice with the development of many various tools (PWA identification for example). Many analysis techniques and/or design methods proposed in the general context of hybrid and switched systems theory may be exploited in the investigation of PWA systems [7,8]. However, most developed ideas do not deal with the general case of PWA systems without (or not common) equilibrium. Indeed, due to the affine term, the absence of common equilibrium is a natural characteristic of PWA systems, and as a consequence, the working point must be chosen among the equilibrium points of the average systems. As this equilibrium point is only virtual, asymptotic stabilization is no longer possible, but only practical stabilization. Consequently, the control is designed so that the trajectory of the closed-loop system can be derived to a small prescribed area enclosing the target point [9-15]. The practical stabilization has been widely investigated, and many important results have been reported for the practical stabilization of low dimensional systems whose attractors are not an equilibrium [9]. For PWA systems, the only available results of specific practical switching stabilization methods are reported in $[12,13]$. The first approach is an old method of Zhai and Michel that has been recently applied to PWA systems in [14]. It is based on the conservative notion of dwell time. The second approach in [13] uses another very conservative condition by requiring a nil average affine term. As a consequence, this can be seen as an extension of the approach in [3,4], with state regions association without removing the revealed principal conservative constraint. Considering a cyclic steady state of PWA systems, another kind of practical switching stabilization method is developed by Reidinger and co-worker in [15]. Independently, the recent proposed approach in [10] is promising due to its simplicity and the absence of any conservative assumption. Moreover, this approach provides the exact distance (error) between the convergence point and the desired working one. A first attempt to complete the above method with multi-estimation technique for bimodal PWA systems was successfully applied to all bimodal DC/DC converters topologies in [11].

Indeed, the design approach in $[10,11]$ is a global asymptotic practical stabilization method for general PWA systems that covers an important class of dynamical systems, with saturation and constraints. More precisely, the main idea consists in finding a suitable state space partition and a state dependent switching law to ensure the overall objective. It is distinguished from the conventional PWA systems stabilization approaches, since it does not assume the existence of common equilibrium and there is not any dwell-time condition on the switching law. Moreover, it is particularly attractive from its closeness to invariance principle, concept that has an important role in asymptotic behaviors of dynamical systems. A positively invariant set is a subset of the state space with the property that, if the system state is at some time in this set, then it remains inside forever [16]. Therefore, the practical stabilization of a system is a sufficient condition for positive invariance since it encloses all reachable states and expresses trajectories boundaries. An important application of this result is in the proof of LaSalle's invariance principle. As a useful tool in the investigation of asymptotic behaviors, various invariance principles and/or extensions of LaSalle's one have been proposed for hybrid and switched systems [16-19]. Furthermore, for linear and/or affine systems, positive invariant sets represent domains of attraction and are generally of simple shapes. However, the switching law may enormously complicate their characterization. In fact, since both continuous and discrete dynamics have to be taken into account for switched systems, an increasing computational effort is required to obtain exact invariants. The boundaries of these templates are characterized by 
exiting sets side and entering ones according to the directions of the flow at these points, so that several invariance based approaches exploit the so-called flow condition.

The concept of invariance is also related to safety property that is generally specified jointly with the formal stability condition. Safety specifications expresses conditions that a system trajectory do not enter a declared unsafe and/or undesired state subset. Its verification is usually translated into a reachability analysis problem that is considered as one of the main addressed problems in the context of hybrid systems. The domain of application is varied, ranging from engineering design to air traffic management systems and biology. Understanding the practical relevance of the problem, lots of research efforts for reachability analysis and verification of hybrid systems have been devoted to the development of implementable systematic procedures. All these developed algorithms should at least approximate the reachable set with safety specifications. Various techniques and methods have emerged to address this problem [19,20]. It is possible to compute explicitly the reachable region and to determine whether the system reaches the unsafe one. However, directly computing the reachable sets is often intractable and requires the system differential equation to be solved. In fact, the efficient computation of reachable sets remains a difficult and open problem. An alternative approach, is to synthesize an invariant for the system i.e. a space region that encloses all reachable states, and then verify the exclusion of the unsafe sets.

Controller design and verification based on invariant sets have been widely investigated particularly in the context of hybrid systems. For state representation, the emphasis is placed on convex approaches where reachable states are usually represented by unions of convex sets (simple shapes).The choice of the representation has great influence on the computation of the underlying sets and on the efficiency of the required operations.

For PWA systems, the need for quadratic invariant was addressed several decades ago [21 and references therein] with ellipsoidal, Cones and Paraboloids templates. Afterward, numerous techniques to synthesize appropriate quadratic templates using SDP solvers and Lyapunov functions are proposed. In spite of the revealed interest of the positive invariance of the individual subsystems in hybrid systems [22], the problem tackled in the present work has for objective to determinate an unique overall positive invariant set for the global PWA system, for which a practical stabilization switching law has been a priori synthesized [10,11]. This approach allows to determine invariant as imposed by the stabilization process and not the converse. One of the objectives of this paper is also to characterize the trajectories $\omega$-limit sets of the global PWA system, in terms of special invariant sets. In other words, subset inclusion tests will not be required anymore, since the underlying set belong to the admissible part of the state space. Due to the importance of the existence of sustained oscillations in the PWA systems design operation, many approaches are devoted to $\omega$-limit set points and their cyclic behaviors. But, nowadays, there is no efficient tool to characterize global stability around limit cycles for PWA systems. The available results in [23] are based on the impact map notion and the newly surfaces Lyapunov functions idea to parameterize switching and to locally study the limit cycles. After some mathematical background and the problem formalization in section II, the main contribution of this paper will be detailed in section III. The proper work will start by determining the $\omega$-limit set points of the positive invariant sets, confirming their periodic and cyclic behaviors. New definitions and mathematical developments will be presented in order to investigate periodic solutions, and the proofs will be based on invariance principle. Section IV presents an extensive simulation results to illustrate the theoretical results. 


\section{Background}

\subsection{Problem formulation}

In this paper, the following PWA models are considered:

$$
\dot{\mathrm{x}}(\mathrm{t})=\mathrm{a}_{\sigma} \mathrm{x}(\mathrm{t})+\mathrm{b}_{\sigma}, t \in \mathrm{R}^{+} .
$$

In which $\mathrm{x}(\mathrm{t}) \in \mathrm{R}^{\mathrm{n}}$ is the state and $\sigma$ is a piecewise constant switching signal: $\sigma(\mathrm{x}): \mathrm{R}^{\mathrm{n}} \rightarrow \mathbb{I}=\{1, \ldots, \mathrm{m}\}$ with $\mathrm{m}$ the finite number of modes in Eq. (1). For state-dependent switching, the system mode activation only depends on the state $\mathrm{x}$, and the target working point $\mathrm{x}_{\mathrm{e}}$ satisfies the following convex combination [2,10]:

$$
\sum_{i=1}^{m} \alpha_{i}\left(a_{i} x_{e}+b_{i}\right)=0,0 \leq \alpha_{i} \leq 1 \text { and } \sum_{i=1}^{m} \alpha_{i}=1 .
$$

In the following, the time variable $t$ is omitted for clarity.

Considering the problem at the origin of (1) and the change of variable $\mathrm{z}=\left[\begin{array}{l}\mathrm{x} \\ 1\end{array}\right]$, a complete synthesis operation was performed in $[10,11]$, where a state space partition and a stabilizing switching law were formulated as a tractable BMI for the augmented following system:

$$
\dot{\mathrm{z}}=\mathrm{A}_{\mathrm{i}} z \text { with } \mathrm{z} \in \mathrm{R}^{\mathrm{n}+1}, \quad \mathrm{~A}_{\mathrm{i}}=\left[\begin{array}{cc}
\mathrm{a}_{\mathrm{i}} & \mathrm{B}_{\mathrm{i}} \\
0 & 0
\end{array}\right], \quad \mathrm{B}_{\mathrm{i}}=\mathrm{a}_{\mathrm{i}} \mathrm{x}_{\mathrm{e}}+\mathrm{b}_{\mathrm{i}} \text { and } \mathrm{i} \in \mathbb{I} .
$$

Let $\dot{\mathrm{V}}_{\mathrm{i}}(\mathrm{z})$ be the time derivative of the common Lyapunov function $\mathrm{V}(\mathrm{z})$ of system (1) along the trajectory of the i-th subsystem, then using the switching law in Eq. (4), the problem has been reduced to the stabilization of system (3) at point: $z^{*}=\left(\begin{array}{llll}0 & \cdots & 0 & 1\end{array}\right)^{t}$.

$$
\sigma(\mathrm{z})=\arg \min _{i \in \mathbb{I}}\left\{\dot{\mathrm{V}}_{\mathrm{i}}(\mathrm{z})\right\} .
$$

where $\sigma(z)=\mathrm{i}$ specifies the mode to be activated.

The idea was based on the following definition [10]: The system (1) is globally asymptotically practically stabilizable by switching at a point $\mathrm{x}_{\mathrm{e}} \in \mathrm{R}^{\mathrm{n}}$, if for every $\varepsilon_{\mathrm{p}} \geq \varepsilon_{\text {pmin }}>0$, there exists $\varepsilon_{\alpha}$ with $0<\varepsilon_{\alpha} \leq \varepsilon_{\mathrm{p}}$ and a switching law that steers the system trajectory from any starting point $\mathrm{x}_{0} \in \mathrm{R}^{\mathrm{n}}$ to the ball $\mathrm{B}\left(\mathrm{x}_{\mathrm{e}}, \varepsilon_{\alpha}\right)$ and maintains it inside $\mathrm{B}\left(\mathrm{x}_{\mathrm{e}}, \varepsilon_{\mathrm{p}}\right)$ for all future time.

The control objectives are then to find the scalars $\varepsilon_{\mathrm{pmin}}, \varepsilon_{\alpha}$ and $\varepsilon_{\mathrm{p}}$.

The design operations for system (3) have been carried out on the following state space subset:

$$
\mathcal{X}=\left\{\mathrm{z} \in \mathrm{R}^{\mathrm{n}+1} / \mathrm{z}_{\mathrm{n}+1}=1\right\} .
$$

The proposed works in $[10,11]$ gave BMI conditions to find a practical stabilizing switching law for the PWA system and the corresponding suitable state space partition, so that all trajectories of the closed-loop system converge to the same domain $\breve{\mathrm{D}} \subset \mathcal{X}$. The invariance of this domain still needs to be proven. In the following subsection, the main mathematical concepts are recalled.

\subsection{Preliminaries}

A quadratic practical stabilization of the augmented system (3) relative to a domain $\breve{D} \subset \mathcal{X}$ can be guaranteed, if there exist a scalar function $\mathrm{V}(\mathrm{z})$, positive scalar $\gamma$ and regions $\mathcal{X}_{\mathrm{i}}$ so that $z^{*} \in \breve{D}$ and 
1) $\mathrm{V}(\mathrm{z})>0$ for all $\mathrm{z \epsilon}\{\mathcal{X}-\breve{\mathrm{D}}\}$.

2) $\dot{\mathrm{V}}_{\mathrm{i}}(\mathrm{z}) \leq-\gamma \mathrm{z}^{\mathrm{t}} z$ for all $z \in \mathcal{X}_{\mathrm{i}}, \mathrm{i} \in \mathbb{I}$.

3) $\cup \mathcal{X}_{\mathrm{i}}=(\mathcal{X}-\breve{\mathrm{D}}), \quad \mathrm{i} \in \mathbb{I}$.

where $\dot{\mathrm{V}}_{\mathrm{i}}(\mathrm{z})$ is the time derivative of $\mathrm{V}(\mathrm{z})$ along the trajectory of the $\mathrm{i}$-th subsystem.

In these approaches, the state space is partitioned in quadratic regions $\Sigma_{i}$, with $\cup \Sigma_{\mathrm{i}}=\mathrm{R}^{\mathrm{n}+1}, \mathrm{i} \in \mathbb{I}$, defined as follows:

$$
\Sigma_{\mathrm{i}}=\left\{z \in \mathrm{R}^{\mathrm{n}+1} \mid \mathrm{z}^{t} \mathrm{Q}_{\mathrm{i}} \mathrm{z} \geq 0\right\}, \quad \mathrm{Q}_{\mathrm{i}}=\left[\begin{array}{cc}
\mathrm{q}_{\mathrm{i}} & \alpha_{\mathrm{qi}} \\
\alpha_{\mathrm{qi}}^{\mathrm{t}} & \beta_{\mathrm{qi}}
\end{array}\right] \in \mathrm{R}^{(\mathrm{n}+1) \times(\mathrm{n}+1)} \text { and } \mathrm{q}_{\mathrm{i}} \in \mathrm{R}^{\mathrm{n} \times \mathrm{n}} .
$$

Two versions of practical stabilization approach have been applied to the system (3).

For a positive symmetric matrix $\mathrm{p} \epsilon \mathrm{R}^{\mathrm{n} \times \mathrm{n}}$ and a positive scalar $\varepsilon_{\mathrm{p}}$, the practical stabilization was first demonstrated for the domain: $\breve{\mathrm{D}}=\left\{z \in \mathcal{X} \mid \mathrm{z}^{\mathrm{t}}\left[\begin{array}{cc}\mathrm{p} & 0 \\ 0 & 0\end{array}\right] \mathrm{z}<\varepsilon_{\mathrm{p}}\right\}, \quad \mathrm{z}^{*} \in \breve{\mathrm{D}}$ after that some refinements of this domain have been made.

- According to Lyapunov theory, the first version in [10] used the following function $\mathrm{V}_{1}(\mathrm{z})$ as energy function for system (3), it is nil at the target point $z^{*}$. i.e. $V_{1}\left(z^{*}\right)=0$.

$$
\mathrm{V}_{1}(\mathrm{z})=\mathrm{z}^{\mathrm{t}} \mathrm{P}_{1} z \text { with } \mathrm{P}_{1}=\left[\begin{array}{cc}
2 \mathrm{p} & \alpha_{\mathrm{p}} \\
\alpha_{\mathrm{p}}^{\mathrm{t}} & 0
\end{array}\right] \in \mathrm{R}^{(\mathrm{n}+1) \times(\mathrm{n}+1)} \text { and } \mathrm{p} \in \mathrm{R}^{\mathrm{n} \times \mathrm{n}}
$$

A value of $\varepsilon_{\alpha}=\varepsilon_{\mathrm{pmin}}=\frac{\alpha_{\mathrm{p}}^{\mathrm{t}} \mathrm{p}^{-1} \alpha_{\mathrm{p}}}{4}$ and a convergence domain $\mathrm{D}_{\alpha 1}$ can be found, according to what is proposed in [10].

More precisely, the used energy function (7) is not positive inside all interior of $\breve{\mathrm{D}}$ :, so that the domain of convergence has been enlarged to $D_{\alpha 1}=\left\{z \in \mathcal{X} \mid z^{t}\left[\begin{array}{ll}p & 0 \\ 0 & 0\end{array}\right] z \leq \alpha_{p}^{t} p^{-1} \alpha_{p}\right\}$.

- In the practical stabilization approach developed in [11], the main idea is based on the fact that the energy of a physical system cannot be nil at an equilibrium point of the average system. According to this principle, the following energy function has been used for system (3).

$$
\mathrm{V}_{2}(\mathrm{z})=\mathrm{z}^{\mathrm{t}} \mathrm{P}_{2} z \text { with } \mathrm{P}_{2}=\left[\begin{array}{cc}
\mathrm{p} & \alpha_{\mathrm{p}} \\
\alpha_{\mathrm{p}}^{\mathrm{t}} & \varepsilon_{\mathrm{p}}
\end{array}\right], i \in \mathbb{I}, \mathrm{P}_{2} \in \mathrm{R}^{(\mathrm{n}+1) \times(\mathrm{n}+1)} \text { and } \mathrm{p} \in \mathrm{R}^{\mathrm{n} \times \mathrm{n}} .
$$

With $\mathrm{V}_{2}\left(\mathrm{z}^{*}\right) \neq 0$. For this strategy, the value of $\varepsilon_{\alpha}=\varepsilon_{\mathrm{pmin}}=\alpha_{\mathrm{p}}^{\mathrm{t}} \mathrm{p}^{-1} \alpha_{\mathrm{p}}$ and a convergence domain $\mathrm{D}_{\alpha}$ can be calculated.

These two results provide two domains of convergence, their associated constraints and the values for $\varepsilon_{\text {pmin }}$. These elements will be used to determinate the invariant sets, and the cyclic nature of the closed-loop behaviors.

\section{Note 1}

If we note by $P_{0}=\left[\begin{array}{cc}p & 0 \\ 0 & 0\end{array}\right], P_{1}=\left[\begin{array}{cc}2 p & \alpha_{p} \\ \alpha_{p}^{t} & 0\end{array}\right]$ and $P_{2}=\left[\begin{array}{cc}p & \alpha_{p} \\ \alpha_{p}^{t} & \varepsilon_{p}\end{array}\right]$ as used in [10,11], and for $\varepsilon \geq 0$, we observe that: 
- All sets defined by $\left\{z \in \mathcal{X} \mid z^{t} P_{0} z=\varepsilon\right\}$ for small positive number $\varepsilon$ are concentric ellipses centered on the average equilibrium $z_{e}$ and are related to the generic energy sets levels of the original PWA system (1), examples are the domains $\breve{D}, D_{\alpha}, D_{\alpha 1}, D_{0}$ and the singleton $z_{e}$ itself.

- All sets defined by $\left\{z \in \mathcal{X} \mid z^{t} P z=\varepsilon\right\}, P=\mathrm{P}_{1}$ or $\mathrm{P}_{2}$ are concentric ellipses centered on $z^{* *}$ and are related to the generic energy sets levels of the augmented system (3), examples are $V_{1}(z)=0, V_{2}(z)=V_{2 \min }(z)$ and $z^{* *}$ itself.

\subsection{Practical switching control strategy}

In these two approaches, the closed-loop switching law consists in stopping the switching as soon as the trajectory reaches the inner domain $\mathrm{D}_{\alpha}$ for the first approach in [10]), and to restart it when the trajectory tries to get out of the outer domain $\breve{\mathrm{D}}$. This technique provides satisfaction and good closed-loop behaviors, it is very useful for the stabilization of general PWA systems. More precisely, it encodes a practical prevention against sliding motions which could occur, by swinging between the two domains, so it reduces the switching frequency when approaching the target point. In fact, the two approach versions rule out of sliding motions because the investigation of such behaviors in the synthesis operation (stabilization by switching) is very difficult see impossible since the regions have to be also found within the design operation.

\subsection{Switched observer}

Due to the state feedback nature of the developed approaches, a Luenberger like-observer has also been proposed for bimodal PWA systems in [11]. The idea was based on the fact that the active mode is indirectly known by the control approach and the studied systems do not present jumps, and so the dynamic of the observer is given by:

$$
\left\{\begin{array}{l}
\dot{\hat{x}}=a_{i} \hat{x}+b_{i}+L_{i}(s-\hat{s}) . \\
\hat{s}=C_{i} \hat{x} .
\end{array}\right.
$$

where $\hat{\mathrm{x}} \in \mathrm{R}^{\mathrm{n}}$, is the estimated states vector and $\mathrm{L}_{\mathrm{i}}$ are the observer gains.

As a result, the dynamic of the estimation error $\tilde{\mathrm{x}}=\mathrm{x}-\hat{\mathrm{x}}$, is also defined by a switched system in Eq. (10). Therefore, the convergence of the estimation error is guaranteed by the stabilization of the corresponding switched system at the origin:

$$
\dot{\tilde{\mathrm{x}}}=\dot{\mathrm{x}}-\dot{\hat{\mathrm{x}}}=\left(\mathrm{a}_{\mathrm{i}}-\mathrm{L}_{\mathrm{i}} \mathrm{C}_{\mathrm{i}}\right) \tilde{\mathrm{x}}, \quad \tilde{\mathrm{x}} \in \bar{\Omega}_{\mathrm{i}}=\left\{\tilde{\mathrm{x}} \in \mathrm{R}^{\mathrm{n}} \mid \tilde{\mathrm{x}}^{t} \overline{\mathrm{q}}_{\mathrm{i}} \tilde{\mathrm{x}} \geq 0\right\} .
$$

Hence, by associating to each individual error a space region $\bar{\Omega}_{\mathbf{i}}$ and using the single candidate Lyapunov function: $\overline{\mathrm{v}}(\tilde{\mathrm{x}})=\tilde{\mathrm{x}} \mathrm{t} \overline{\mathrm{x}}, \overline{\mathrm{p}}=\overline{\mathrm{p}}^{\mathrm{t}}>0$, the stabilizing conditions of system (10) are formulated as NMI (Nonlinear Matrix Inequality). With a change of variable and the help of S-Procedure, the conditions are transformed to tractable BMI (Bilinear Matrix Inequality) and the observer gains are calculated. With some synchronization process between switching instants control and multi-observer states update, the method can easily be applied to the general PWA systems with $\mathrm{n}$ modes. The results are exploited to compute the period of oscillations in the next section.

Recall, an invariant is a property that holds at all reachable states from any initial state that satisfies this property. Therefore, an interesting additional investigation consists in knowing 
whether the same domain of convergence is maintained when the observer pilots the system, and when it starts from the same or different initials conditions of the system. This fact is explored by the simulation results in Fig. 2. Where, the convergence is proved to be toward $\mathrm{z}^{* *}$ even if starting from the target point $\mathrm{z}^{*}$, see Fig. 2-c.

\section{PWA systems: invariant sets and limit set points}

Invariant sets play an important role in systems control theory [16-22]; they are known as sets containing all trajectories when starting inside, the simplest ones are the level sets of Lyapunov functions. However, this notion cannot be directly extended to PWA systems for which a state space partition and switching control strategy must be specified. Another important notion is the $\omega$-limits set points, which are defined as the convergence set points after an infinitely time trajectory evolution. They also have to be readapted for switched systems. Indeed, for such systems, the asymptotic behavior defined by some set points limits cannot be distinguished without specifying the switching law for which the set is captured.

The stability notion for switched systems under arbitrary switching signal, is the only notion that may lead to the uniformly invariance set property. However, this property is very conservative and often unfeasible for real cases. For instance, we often need state space partition, multiple Lyapunov functions or dwell-time property to stabilize or to prove the stability of switched systems, but all these tools do not deal with uniformity. Here are some proposed rearrangements of these definitions:

Definition 1. For a switched PWA system $\dot{x}(t)=a_{i} x(t)+b_{i}, t \in R^{+}$and $i \in \mathbb{I}$, a set $\mathrm{M}$ is said to be an uniformly invariant set, if for all $x_{0 i} \in M, \quad i \in \mathbb{I}$ and for any switching signal, the trajectory $x_{j}(t) \in M, \quad j \in \mathbb{I}$ and then $x(t) \in M$.

Definition 2. For a switched PWA system $\dot{x}(t)=a_{i} x(t)+b_{i}, t \epsilon R^{+}$and $i \in \mathbb{I}$, a set $\mathrm{M}$ is said to be an invariant set with respect to a switching signal $\sigma(\mathrm{x})$, if for all $x_{0 i} \in M, \quad i \in \mathbb{I}$ and this switching signal, the trajectory $x_{j}(t) \in M, \quad j \in \mathbb{I}$ and then $x(t) \in M$.

Based on the same ideas, asymptotic behaviors and sets $\omega$-limit points may be defined as follow:

Definition 3. For a switched PWA system $\dot{x}(t)=a_{i} x(t)+b_{i}, t \epsilon R^{+}$and $i \in \mathbb{I}$, a set $\omega$ represents an $\omega$-limit set points with respect to a switching signal $\sigma(\mathrm{x})$, if for all initial condition $x_{0 i}, \quad i \in \mathbb{I}$ and this switching signal, the trajectory $x(t)$ approaches $\omega$ as $\mathrm{t} \rightarrow+\infty$.

As the analyses depend on the switching law, the following section will be based on the two stabilizing strategies developed in $[10,11]$.

\subsection{Main results}

The D domain, obtained during the stabilization is as invariant subset, centered on $\mathrm{z}^{*}$. Therefore, this outer convergence domain may be considered as a starting point for our investigation. The inner convergence domains $D_{\alpha 1}$ in [10] and $D_{\alpha}$ in [11] will be used to determine the associated $\omega$-limit set points for the switched augmented system (3). However, 
these domains are results of enlargements and adjustments, hence they are not directly related to theirs corresponding $\omega$-limit set points. Instead of the direct use of these domains, the results will be based on theirs corresponding $\varepsilon_{\min }$ and $\mathrm{V}(\mathrm{z})_{\min }$.

Let us consider the problem at the origin, then the reference point $\mathrm{z}_{\mathrm{e}}$ becomes $\mathrm{z}^{*}=(0 \ldots 01)^{t} \in \check{\mathrm{D}}$, this implies that the invariant subset $\check{\mathrm{D}}$ contains at least one average equilibrium point. More precisely, an $\omega$-limit set points cannot be empty inside an invariant subset and cannot be reduced to a singleton since there is no common equilibrium. On the other hand, for the two proposed approaches, it was shown that all trajectories try to converge toward $\mathrm{z}^{* *}$ and are maintained around by switching which makes oscillations appear. Finally, unproven statements of several works attest that these systems can exhibit cyclic behaviors around the average equilibrium. We propose here to exhibit the conditions under which these cyclic behaviors occur.

Theorem 1. Let us consider a PWA system $\dot{x}(t)=a_{i} x(t)+b_{i}$ without common equilibrium $i \in \mathbb{I}_{m}, \quad x(t) \in R^{n}$ and $t \in R^{+}$. For an energetic function $V(z), \quad z=\left[\begin{array}{l}x \\ 1\end{array}\right]$ and for some stabilizing switching control $\sigma(\mathrm{x})$, let $M$ be a positive invariant subset with respect to $\sigma(\mathrm{x})$, that contains at least one point $z_{e}=\left[\begin{array}{c}x_{e} \\ 1\end{array}\right]$ with $x_{e}$ average equilibrium point of the PWA system, then $\forall \mathrm{p} \in \mathrm{M}$, the set $\omega$-limit points verifies:

1) $\omega(p)$ is a non-empty set that cannot be reduced to a singleton.

And one of the two following assertions is true:

2) $\omega(p)$ is a stable quasi-periodic orbit around $z^{* *} \neq z_{e}$ with $z^{* *}=\arg _{z \in M}\left(V_{\min }(z)\right)$ and $d\left(z^{* *}, z_{e}\right)$

very small calculable error.
$\omega(p)$ is a stable periodic orbit around $z_{e}=\left[\begin{array}{c}x_{e} \\ 1\end{array}\right]$.

\subsection{Detailed mathematical proof of theorem 1}

Let us note $\mathcal{E}_{\alpha \omega} \subset \mathrm{D}_{\alpha}$ and $\mathcal{E}_{\alpha 1 \omega} \subset \mathrm{D}_{\alpha 1}$ respectively the two $\omega$-limit set points in [10,11]. The proof is accomplished when these sets are accurately designed as closed and non-empty connected subsets, the periodicity and stability of the infinite time trajectories evolution (limit cycles) is established, and the associated critical parameters calculated.

\subsubsection{Design of $\omega$-limit sets points}

- For the second approach version [11], no restriction for the trajectory to reach $\mathrm{z}^{* *}$ but with some quantity of energy and the corresponding $\omega$-limit set points may reduce to one point that is $\mathrm{z}^{* *}$. Nevertheless, the trajectory cannot be maintained at this point for two reasons:

i. Because $\mathrm{z}^{* *}$ is not a common equilibrium and enforced switching is needed to move toward it. ii. If reached, this is done with a not nil energy i.e. $\mathrm{V}_{2 \min }(\mathrm{z})=\mathrm{V}\left(\mathrm{z}^{* *}\right)=\varepsilon_{\mathrm{p}}-\alpha_{\mathrm{p}}^{\mathrm{t}} \mathrm{p}^{-1} \alpha_{\mathrm{p}} \neq 0$ for $\mathrm{z}^{* *}$ $=\left(\begin{array}{c}\mathrm{x}^{* *} \\ 1\end{array}\right)$ with $\mathrm{x}^{* *}=-\mathrm{p}^{-1} \alpha_{\mathrm{p}}$. In this case, the corresponding $\omega$-limit set points are given by 
the closure of the following set:

$$
\mathcal{E}_{\alpha \omega}=\left\{\mathrm{z} \in \mathcal{X} \mid \mathrm{z}^{\mathrm{t}}\left[\begin{array}{cc}
\mathrm{p} & \alpha_{\mathrm{p}} \\
\alpha_{\mathrm{p}}^{\mathrm{t}} & \varepsilon_{\mathrm{p}}
\end{array}\right] \mathrm{z} \leq \varepsilon_{\mathrm{p}}-\alpha_{\mathrm{p}}^{\mathrm{t}} \mathrm{p}^{-1} \alpha_{\mathrm{p}}\right\}=\left\{\mathrm{z} \in \mathcal{X} \mid \mathrm{z}^{\mathrm{t}}\left[\begin{array}{cc}
\mathrm{p} & \alpha_{\mathrm{p}} \\
\alpha_{\mathrm{p}}^{\mathrm{t}} & \alpha_{\mathrm{p}}^{\mathrm{t}} \mathrm{p}^{-1} \alpha_{\mathrm{p}}
\end{array}\right] \mathrm{z} \leq 0\right\}
$$

This represents a reduction of an ellipsoid to one point; while the enforced switching tries to maintain the trajectory at this point, the results are oscillations around $\mathrm{z}^{* *}$.

For the above reasons, the existence of oscillations is guaranteed. However, to make their appearance more clearly, we must eliminate the possibility of the reduction of the ellipsoid closure that represents the $\omega$-limit set points as follow:

Since $\varepsilon_{\mathrm{p}} \geq \alpha_{\mathrm{p}}^{\mathrm{t}} \mathrm{p}^{-1} \alpha_{\mathrm{p}}>0$, we have for any $z \in \mathcal{X}$

$$
\begin{aligned}
\mathrm{z}^{\mathrm{t}}\left[\begin{array}{cc}
\mathrm{p} & \alpha_{\mathrm{p}} \\
\alpha_{\mathrm{p}}^{\mathrm{t}} & \varepsilon_{\mathrm{p}}
\end{array}\right] \mathrm{z} \leq \varepsilon_{\mathrm{p}}-\alpha_{\mathrm{p}}^{\mathrm{t}} \mathrm{p}^{-1} \alpha_{\mathrm{p}} & \Longrightarrow \mathrm{z}^{\mathrm{t}}\left[\begin{array}{cc}
\mathrm{p} & \alpha_{\mathrm{p}} \\
\alpha_{\mathrm{p}}^{\mathrm{t}} & \varepsilon_{\mathrm{p}}
\end{array}\right] \mathrm{z} \leq \varepsilon_{\mathrm{p}}-\delta \alpha_{\mathrm{p}}^{\mathrm{t}} \mathrm{p}^{-1} \alpha_{\mathrm{p},} \delta \leq 1 \\
& \Longrightarrow \mathrm{z}^{\mathrm{t}}\left[\begin{array}{cc}
\mathrm{p} & \alpha_{\mathrm{p}} \\
\alpha_{\mathrm{p}}^{\mathrm{t}} & \delta \alpha_{\mathrm{p}}^{\mathrm{t}} \mathrm{p}^{-1} \alpha_{\mathrm{p}}
\end{array}\right] \mathrm{z} \leq 0
\end{aligned}
$$

In order that the last term represents a non-empty ellipsoid, $\delta$ must be strictly less than 1 .

In fact, the introduction of the parameter $\delta$ allows ingeniously depriving the energy function $\mathrm{V}(\mathrm{z})$ to reach its minimum $\mathrm{V}_{2 \min }(\mathrm{z})=\mathrm{V}\left(\mathrm{z}^{* *}\right)=\varepsilon_{\mathrm{p}}-\alpha_{\mathrm{p}}^{\mathrm{t}} \mathrm{p}^{-1} \alpha_{\mathrm{p}}$ and stop the convergence at slightly higher value $\tilde{V}_{2 \min }(\mathrm{z})=\varepsilon_{\mathrm{p}}-\delta \alpha_{\mathrm{p}}^{\mathrm{t}} \mathrm{p}^{-1} \alpha_{\mathrm{p}}$.

For accuracy reason, $\delta$ must be positive and very near the unity say $0 \ll \delta<1$ and it may be used as a free parameter.

The new $\omega$-limit set points for the second method is given by the closure of the following ellipsoid:

$$
\tilde{\varepsilon}_{\alpha \omega}=\left\{z \in \mathcal{X} \mid z^{t}\left[\begin{array}{cc}
p & \alpha_{p} \\
\alpha_{p}^{t} & \delta \alpha_{p}^{t} p^{-1} \alpha_{p}
\end{array}\right] z \leq 0\right\}
$$

Notice this ellipsoid representation is homogenous; the multiplication of the inner matrix by any positive factor does not affect the ellipsoid. In order to make appear the center, and since the $(\mathrm{n}+1)$-th element composing $\mathcal{X}$ is constant (equal to 1$)$ then this description may be identified to the following representation in $\mathrm{R}^{\mathrm{n}}$ :

$$
\tilde{\mathcal{E}}_{\alpha \omega}=\left\{\mathrm{x} \in \mathrm{R}^{\mathrm{n}}\left(\mathrm{x}-\mathrm{x}_{\mathrm{c}}\right)^{\mathrm{t}} \mathrm{N}^{-1}\left(\mathrm{x}-\mathrm{x}_{\mathrm{c}}\right) \leq 1\right\}, \quad \mathrm{N}^{\mathrm{t}}=\mathrm{N}>0
$$

That can be rewritten in $\mathcal{X}$ as follow:

$$
\tilde{\varepsilon}_{\alpha \omega}=\left\{\mathrm{z} \in \mathcal{X} \mid \mathrm{z}^{\mathrm{t}}\left[\begin{array}{cc}
\mathrm{N}^{-1} & -\mathrm{N}^{-1} \mathrm{x}_{\mathrm{c}} \\
-\mathrm{x}_{\mathrm{c}}^{\mathrm{t}} \mathrm{N}^{-1} & x_{\mathrm{c}}^{\mathrm{t}} \mathrm{N}^{-1} x_{\mathrm{c}}-1
\end{array}\right] \mathrm{z} \leq 0\right\}
$$

We deduce by identification:

$$
\left\{\begin{array}{c}
N=\mathrm{p}^{-1} \\
\mathrm{x}_{\mathrm{c}}=-\mathrm{p}^{-1} \alpha_{\mathrm{p}}
\end{array} \text { and } \mathrm{x}_{\mathrm{c}}^{\mathrm{t}} \mathrm{N}^{-1} \mathrm{x}_{\mathrm{c}}-1=\delta \alpha_{\mathrm{p}}^{\mathrm{t}} \mathrm{p}^{-1} \alpha_{\mathrm{p}}\right.
$$


From the last equation, we obtain: $(1-\delta) \alpha_{\mathrm{p}}^{\mathrm{t}} \mathrm{p}^{-1} \alpha_{\mathrm{p}}=1$, this condition can be used to scale the ellipsoid equation leading to:

$$
\tilde{\varepsilon}_{\alpha \omega}=\left\{z \in \mathcal{X} \mid z^{t}\left[\begin{array}{cc}
p & \alpha_{p} \\
\alpha_{p}^{t} & \alpha_{p}^{t} p^{-1} \alpha_{p}-1
\end{array}\right] z \leq 0\right\}
$$

This ellipsoid is still centered on $\mathrm{z}^{* *}=\left(\begin{array}{c}\mathrm{x}^{* *} \\ 1\end{array}\right)=\left(\begin{array}{c}\mathrm{x}_{\mathrm{c}} \\ 1\end{array}\right)$ with $\mathrm{x}^{* *}=\mathrm{x}_{\mathrm{c}}=-\mathrm{p}^{-1} \alpha_{\mathrm{p}}$ where the lengths of its semi- axis are given by the square roots of the scaled matrix, e.g. the maximum semi-axis length is:

$$
\mathrm{d}_{g \alpha \mathrm{M}}=\sqrt{\alpha_{\mathrm{p}}^{\mathrm{t}} \mathrm{p}^{-1} \alpha_{p}(1-\delta) \lambda_{\max }\left(\mathrm{p}^{-1}\right)}
$$

Using $\delta$ as a user-made parameter with $0 \ll \delta<1$, we have the following result:

$$
\begin{gathered}
\lambda_{\max }\left(\mathrm{p}^{-1}\right)=\frac{\mathrm{d}_{g \alpha \mathrm{M}}{ }^{2}}{\alpha_{\mathrm{p}}^{\mathrm{t}} \mathrm{p}^{-1} \alpha_{p}(1-\delta)} \Longrightarrow \frac{1}{\lambda_{\min }(\mathrm{p})}=\frac{\mathrm{d}_{g \alpha \mathrm{M}}{ }^{2}}{\alpha_{\mathrm{p}}^{\mathrm{t}} \mathrm{p}^{-1} \alpha_{p}(1-\delta)} \\
\Longrightarrow \lambda_{\min }(\mathrm{p})=\frac{\alpha_{\mathrm{p}}^{\mathrm{t}} \mathrm{p}^{-1} \alpha_{p}(1-\delta)}{\mathrm{d}_{g \alpha \mathrm{M}}{ }^{2}} \\
\Longrightarrow \mathrm{p}>\frac{\alpha_{\mathrm{p}}^{\mathrm{t}} \mathrm{p}^{-1} \alpha_{p}(1-\delta)}{\mathrm{d}_{g \alpha \mathrm{M}}{ }^{2}}
\end{gathered}
$$

Since $\varepsilon_{\mathrm{p}} \geq \alpha_{\mathrm{p}}^{\mathrm{t}} \mathrm{p}^{-1} \alpha_{\mathrm{p}}$ which must be minimized in the LMI optimization of theorem 1 in [11], then $\alpha_{\mathrm{p}}^{\mathrm{t}} \mathrm{p}^{-1} \alpha_{\mathrm{p}}$ can be replaced by $\varepsilon_{\mathrm{p}}$ and the condition to be included in theorem 1 in [11] to ensure an $\omega$-limit set points as an ellipsoid with a prescribed maximum axis length $\mathrm{d}_{g \alpha \mathrm{M}}$ is given by:

$$
\mathrm{p}>\frac{(1-\delta) \varepsilon_{\mathrm{p}}}{\mathrm{d}_{g \alpha \mathrm{M}}{ }^{2}}
$$

- In the first version [10], the $\omega$-limit set points is described by $V_{1}(z)=z^{t}\left[\begin{array}{cc}2 p & \alpha_{p} \\ \alpha_{p}^{t} & 0\end{array}\right] z=0$, since the trajectory can't continue (converges) to $\mathrm{z}^{* *}$ because the used energy function is not positive inside see Section 2.2 for more detail. So that, the $\omega$-limit points are directly given by the closure of the following ellipsoid:

$$
\varepsilon_{\alpha 1 \omega}=\left\{\mathrm{z} \in \mathcal{X} \mid z^{t}\left[\begin{array}{cc}
2 \mathrm{p} & \alpha_{\mathrm{p}} \\
\alpha_{\mathrm{p}}^{\mathrm{t}} & 0
\end{array}\right] \mathrm{z} \leq 0\right\}
$$
As before, this non-empty ellipsoid is centered on $\mathrm{z}^{* *}=\left(\begin{array}{c}\mathrm{x}^{* *} \\ 1\end{array}\right)$ with $\mathrm{x}^{* *}=-\frac{\mathrm{p}^{-1} \alpha_{\mathrm{p}}}{2}$ and a
maximum semi-axis length (from scaled matrix):

$$
\mathrm{d}_{g \alpha 1 \mathrm{M}}=\frac{\sqrt{\left(\alpha_{\mathrm{p}}^{\mathrm{t}} \mathrm{p}^{-1} \alpha_{p}\right) \lambda_{\max }\left(\mathrm{p}^{-1}\right)}}{2}
$$


Then the condition to be included in the theorem 1 in [10] of the first approach version is given by:

$$
\mathrm{p}>\frac{\varepsilon_{\mathrm{p}}}{4 \mathrm{~d}_{\mathrm{g} \alpha \mathrm{M}}{ }^{2}} .
$$

Note 2

Since $\mathcal{E}_{\alpha \omega}(\mathrm{z})$ is just a representation of $\mathcal{E}_{\alpha \omega}(\mathrm{x})$ in the augmented space with a constant (equal to 1) of the $(n+1)^{\text {th }}$ dimension of $z$, we do not distinguish $\varepsilon_{\alpha \omega}(\mathrm{z})$ from $\mathcal{E}_{\alpha \omega}(\mathrm{x})$ when speaking about the $\omega$-limit set points $\varepsilon_{\alpha \omega}$.

\subsubsection{Periodicity of the infinite time trajectories evolution}

It is demonstrated that for the stated practical stabilizing conditions and when starting from any initial point, all trajectories of system (3) approach the closed and non-empty connected subset $\varepsilon_{\alpha \omega}$ (or $\varepsilon_{\alpha 1 \omega}$ ) which is also a path-connected subset. Furthermore, this $\omega$-limit set points does not contain any equilibrium and no trajectory can enter this domain from outside coming, and reciprocally no trajectories can escape it when starting inside (see Fig. 3). In that case, it is not difficult to find a translation number $\tau(\epsilon)$ for any positive real number $\epsilon$ so that:

$$
\|\mathrm{x}(\mathrm{t}+\tau(\epsilon))-\mathrm{x}(\mathrm{t})\| \leq \epsilon \text { when } x(\mathrm{t}) \text { approaches these } \omega \text { - limit set points. }
$$

These trajectories cannot be other than periodic or quasi-periodic evolution which is characterized by the above propriety.

Recall, a trajectory $\mathrm{x}(\mathrm{t})$ will be called a limits cycle when its distance to the $\omega$-limit set points $\varepsilon_{\alpha \omega}$ vanishes for infinite time evolution i.e.

$$
\lim _{\mathrm{t} \rightarrow+\infty} \mathrm{d}\left(\mathrm{x}(\mathrm{t}), \varepsilon_{\alpha \omega}\right)=0
$$

\subsubsection{Trajectories stability \\ We have the following result:}

Proposition 1. Under practical stabilizing conditions, all trajectories of system (3) end up by stable periodic or quasi-periodic orbits.

The proof is indirectly provided by the fact that our practical stabilization approaches are demonstrated and validated for all trajectories and these periodic evolutions are simple trajectories then these periodic or quasi-periodic orbits are certainly stables. Using an extension of the small-gain theorem for general nonlinear systems, the robustness of the existence of periodic trajectories has already been studied before in [24].

\subsubsection{Periodic trajectories parameters calculation}

In plus of the amplitude, the period of oscillations is a critical parameter that must be calculated or approximated for periodic trajectories. In our case, many interesting informations are available so that any numerical method based sampling time at switching instants may be used to obtain the period $[25,26]$. A good approximation of this parameter suffices since it is not used in the analysis nor for the synthesis, nevertheless, precise techniques can be used for informative purpose. 


\section{i. Period calculation}

Fortunately, our approach is equipped with an efficient on-line general state estimation technique. The subsystem (mode) to be activated is provided by the control approach at each instant time. Then a time counter can suffice to determine the period of oscillations, moreover, all switching instants may be identified. However, because of the global nature of the proved stabilization approach, the trajectory may start far away and makes time to reach the $\omega$-limit set points $\varepsilon_{\alpha \omega}$. So that, a judicious initialization of the time counter is necessary.

Let $\hat{t}_{0}$ be the first time the trajectory reaches exactly the closure of $\varepsilon_{\alpha \omega}$, this will be detected by the fulfillment of the following condition:

$$
\varepsilon_{\alpha \omega}\left(\hat{\mathrm{z}}\left(\hat{\mathrm{t}}_{0}\right)\right)=0
$$

Let $t_{0} \geq \hat{t}_{0}$ be the time at which the first switch on the closure of $\varepsilon_{\alpha \omega}$ occurs.

Let $\mathrm{M}_{\mathrm{k}}, \quad \mathrm{k}=\{1, \ldots, \mathrm{m}\}$ be three columns matrices, $\mathrm{M}_{\mathrm{k}}=\left[\mathrm{T}_{\mathrm{M}}^{\mathrm{k}} \mathrm{v}^{\mathrm{k}} \mathrm{u}^{\mathrm{k}}\right] \quad$ with $\mathrm{T}_{\mathrm{M}}^{\mathrm{k}}=\left(\begin{array}{c}\mathrm{t}_{1}^{\mathrm{k}} \\ \vdots \\ \mathrm{t}_{\mathrm{n}_{\mathrm{k}}}^{\mathrm{k}}\end{array}\right), \quad \mathrm{v}^{\mathrm{k}}=\left(\begin{array}{c}\left(\mathrm{y}_{1}^{\mathrm{k}}\right)^{\mathrm{t}} \mathrm{y}_{1}^{\mathrm{k}} \\ \vdots \\ \left(\mathrm{y}_{\mathrm{n}_{\mathrm{k}}}^{\mathrm{k}}\right)^{\mathrm{t}} \mathrm{y}_{\mathrm{n}_{\mathrm{k}}^{\mathrm{k}}}^{\mathrm{k}}\end{array}\right)$ and $\mathrm{u}^{\mathrm{k}}=\left(\begin{array}{c}\left(\hat{\mathrm{x}}_{1}^{\mathrm{k}}\right)^{\mathrm{t}} \hat{\mathrm{x}}_{1}^{\mathrm{k}} \\ \vdots \\ \left(\hat{\mathrm{x}}_{\mathrm{n}_{\mathrm{k}}}^{\mathrm{k}}\right)^{\mathrm{t}} \hat{\mathrm{x}}_{\mathrm{n}_{\mathrm{k}}}^{\mathrm{k}}\end{array}\right)$.

Let $\mathrm{R}_{\mathrm{k}}, \mathrm{k}=\{1, \ldots, \mathrm{m}\} \quad$ be two columns matrices, $\mathrm{R}_{\mathrm{k}}=\left[\mathrm{T}_{\mathrm{R}}^{\mathrm{k}} \mathrm{s}^{\mathrm{k}}\right]$ with $\mathrm{T}_{\mathrm{R}}^{\mathrm{k}}=\left(\begin{array}{c}\tau_{1}^{\mathrm{k}} \\ \vdots \\ \tau_{\mathrm{n}_{\mathrm{k}}}^{\mathrm{k}}\end{array}\right), s^{\mathrm{k}}=\left(\begin{array}{c}\left(\hat{\mathrm{x}}_{1}^{\mathrm{k}}\right)^{\mathrm{t}} \hat{\mathrm{x}}_{1}^{\mathrm{k}} \\ \vdots \\ \left(\hat{\mathrm{x}}_{\mathrm{n}_{\mathrm{k}}}^{\mathrm{k}}\right)^{\mathrm{t}} \hat{\mathrm{x}}_{\mathrm{n}_{\mathrm{k}}^{\mathrm{k}}}^{\mathrm{k}}\end{array}\right)$.

With $\hat{x}_{i}^{k}$ is the estimated state during mode $\mathrm{k}$ at time $\mathrm{t}_{\mathrm{i}}^{\mathrm{k}}$, and $\mathrm{y}_{\mathrm{i}}^{\mathrm{k}}=\hat{\mathrm{x}}_{\mathrm{i}}^{\mathrm{k}}-\mathrm{x}_{\mathrm{c}}$. Notice that $\mathrm{t}_{1}^{\mathrm{k}} \geq \mathrm{t}_{0}$ is the first activation time of mode $\mathrm{k}$ on the closure of $\varepsilon_{\alpha \omega}$ and $\hat{\mathrm{x}}_{1}^{\mathrm{k}}$ is the corresponding estimated state. Whereas, $\mathrm{t}_{\mathrm{n}_{\mathrm{k}}}^{\mathrm{k}}$ is the deactivation time of mode $\mathrm{k}$ on the closure of $\varepsilon_{\alpha \omega}$, and $\hat{\mathrm{x}}_{\mathrm{n}_{\mathrm{k}}}^{\mathrm{k}}$ is the corresponding estimated state. Obviously, the matrices $\mathrm{M}_{\mathrm{k}}$ have different dimensions since the activation period are not necessary equal, and same remark for $\mathrm{R}_{\mathrm{k}}$.

Without loss of generality, let us suppose that all modes are visited and in the numerical order $\mathrm{k}=1, k=2, \ldots \ldots \mathrm{k}=\mathrm{m}$ then the state continuity of PWA systems leads to:

$$
\mathrm{t}_{\mathrm{n}_{\mathrm{k}}}^{\mathrm{k}}=\mathrm{t}_{1}^{\mathrm{k}+1}
$$

Taking $\mathrm{t}_{1}^{1}=\tau_{1}^{1}=\mathrm{t}_{0}$, this means that at time $\mathrm{t}_{\mathrm{m}}=\mathrm{t}_{\mathrm{n}_{\mathrm{m}}}^{\mathrm{m}}=\tau_{\mathrm{n}_{\mathrm{m}}}^{\mathrm{m}}$ all modes are visited each once. Henceforth, we stop updating the matrices $\mathrm{M}_{\mathrm{k}}$ that must be stored and we keep updating the matrices $R_{k}$ without saving $R_{k-1}$. In other words, at time $t_{m}$, another cycle of modes succession starts. After each mode evolution the following vector can be computed:

$$
\mathrm{w}^{\mathrm{k}}=\left(\mathrm{s}^{\mathrm{k}}-\mathrm{u}^{\mathrm{k}}\right)
$$


If $\mathrm{w}^{\mathrm{k}}=0$ then $\mathrm{s}^{\mathrm{k}}=\mathrm{u}^{\mathrm{k}}$ which means that:

$$
\left(\hat{\mathrm{x}}_{\mathrm{i}}^{\mathrm{k}}\left(\mathrm{t}_{\mathrm{i}}\right)\right)^{\mathrm{t}} \hat{\mathrm{x}}_{\mathrm{i}}^{\mathrm{k}}\left(\mathrm{t}_{\mathrm{i}}\right)=\left(\hat{\mathrm{x}}_{\mathrm{i}}^{\mathrm{k}}\left(\tau_{\mathrm{i}}\right)\right)^{\mathrm{t}} \hat{\mathrm{x}}_{\mathrm{i}}^{\mathrm{k}}\left(\tau_{\mathrm{i}}\right), \mathrm{i}=1, \mathrm{n}_{\mathrm{k}} \text { and } \tau_{\mathrm{i}} \geq \mathrm{t}_{\mathrm{m}}>\mathrm{t}_{\mathrm{i}}
$$

Since $\varepsilon_{\alpha \omega}$ is centered on $x^{* *}=x_{c}=-p^{-1} \alpha_{p} \neq x^{*}=\left(\begin{array}{c}0 \\ \vdots \\ 0\end{array}\right)$; it can be shown that $\mathrm{x} \in \mathcal{E}_{\alpha \omega} \nRightarrow-\mathrm{x} \in \mathcal{E}_{\alpha \omega}$ and we have the following result:

$$
\text { If } \mathrm{w}^{\mathrm{k}}=0 \Rightarrow \hat{\mathrm{x}}_{\mathrm{i}}^{\mathrm{k}}\left(\mathrm{t}_{\mathrm{i}}\right)=\hat{\mathrm{x}}_{\mathrm{i}}^{\mathrm{k}}\left(\tau_{\mathrm{i}}\right), i=1, \mathrm{n}_{\mathrm{k}} \text { and } \tau_{\mathrm{i}} \geq \mathrm{t}_{\mathrm{m}}>\mathrm{t}_{\mathrm{i}}
$$

Then the period of trajectory oscillations is given by:

$$
\mathrm{T}_{\mathrm{p}}=\mathrm{T}_{\mathrm{R}}^{\mathrm{k}}(\mathrm{i})-\mathrm{T}_{\mathrm{M}}^{\mathrm{k}}(\mathrm{i})=\tau_{1}^{\mathrm{k}}-\mathrm{t}_{1}^{\mathrm{k}}
$$

\section{ii. Amplitude calculation}

Even if the precious information is calculated i.e. the ellipsoid maximum axis length $\mathrm{d}_{g \alpha \mathrm{M}}$ which is related in temporal domain to the maximum amplitude of oscillation; it is also possible to determine this parameter from the above numerical on-line algorithm as follow:

The maximum amplitude of oscillation can be computed directly from the stored matrices since these data are collected after the trajectory arrival to the $\omega$-limit set points (the cyclic part). If we denote by $\mathrm{a}_{\max }$ this maximum amplitude, we have:

$$
\mathrm{a}_{\max }=\max _{1 \leq \mathrm{k} \leq \mathrm{m}} \max _{1 \leq \mathrm{i} \leq \mathrm{n}_{\mathrm{k}}}\left(\operatorname{sqrt}\left(\mathrm{v}^{\mathrm{k}}(\mathrm{i})\right)\right)
$$

This mathematical detail completes the proof of Theorem 1 .

\subsubsection{Computational complexities and remarks}

1. The conditions in the three cited theorems are BMI, they could not be directly tackled by classic software before a gridding up operation of unknown scalars variables, this give an extra degrees of freedom in the computation. Moreover, the BMI conditions cover the corresponding LMI ones as special cases, hence theirs results are guaranteed to be at least as good as the LMI ones. Nevertheless, LMI Matlab toolbox is used to perform our simulations on the transformed BMI. Besides this note, the same notation has been used for the two approaches in $[10,11]$ but after optimization $\mathrm{p}, \alpha_{p}$ and $\varepsilon_{p}$ are not necessary the same.

2. Automatic calculation and simplification may be obtained in the second approach version by setting the instrumental parameter $\delta=0$, but this will slightly affect the results accuracy by increasing the diameter of the target ellipsoid.

3. It may be difficult to determine exactly the time instants $t_{0}$ and $t_{m}$, this is not crucial since $t_{0}$ may be chosen as any one among the switching times on $\varepsilon_{\alpha \omega}$ and not necessary the first one, whenever condition (24) is crucial. Similarly, $t_{m}$ may be equal to any one of switching times of the second or other cycle but necessary at least one cycle after $t_{0}$.

4. The condition (24) may not be satisfied or takes a very long evolution time to be verified, a relaxation consist to use in place: $\varepsilon_{\alpha \omega}\left(\hat{\mathrm{Z}}\left(\hat{\mathrm{t}}_{0}\right)\right)=\mu$, with $\mu$ a very small positive number according to the working machine precision, and similarly for $\mathrm{w}^{\mathrm{k}}=0$. 
5. The number of visited modes is crucial to mark the end of cycle $\left(\mathrm{t}_{\mathrm{n}_{\mathrm{m}}}^{\mathrm{m}}\right.$ or $\left.\tau_{\mathrm{n}_{\mathrm{m}}}^{\mathrm{m}}\right)$. A preliminary simulation test may be necessary to facilitate the determination of the number of visited modes and theirs succession when $\varepsilon_{\alpha \omega}$ is reached.

Note 3

In the absence of common equilibrium for PWA systems, there is no strict convergence towards the average equilibrium $z^{*}$. However, the convergence toward $z^{* *}$ is not proved as inherent property of PWA systems, it may be just an effect of the working state space augmentation.

\section{Persevering issues in the stabilization of PWA systems}

In spite of the PWA functions versatility, ease of implementation and approximation of most nonlinear behaviors, PWA system with fixed parameters might not provide a good model of a real system. Since in practice, there are important and inevitable factors that are often neglected in performance analysis and control synthesis, leading to significant discrepancies between the real behavior of the system and what are expected from simulations. For examples, parametric uncertainty and time delay are of great importance in real situations. Moreover, the time delay may be variable and/or uncertain without known ranges [27,28]. Taking account these factors in the design control of general PWA systems may considerably complicate the operation, where parameter uncertainty and/or time delay have to be strictly specified for each subsystem. Since theirs primarily role in instability and poor performances for controlled systems is currently well recognized [27], then neglecting these factors or theirs effects is also not a good solution. Moreover, the phenomena of time delay in PWA and switched systems may be very complicated. In fact, there may also exist time delay between modes switching and control process switching, this often occurs when the switching control process makes more time to act on the real plant. Examples of these problems may be encountered in all networked control systems communicating by channels. This will lead to asynchronous switching that may also destabilize the controlled system [29]. Similarly, asynchronous switching can appear between plant modes switching and the observer ones when multi-estimation technique is employed as the one presented in this work, then destroyed estimation results are to be expected. Although, switched systems with time delay have received great interest [27], few works are devoted to PWA ones [30], and more recently, many researchers commence addressing asynchronous

a

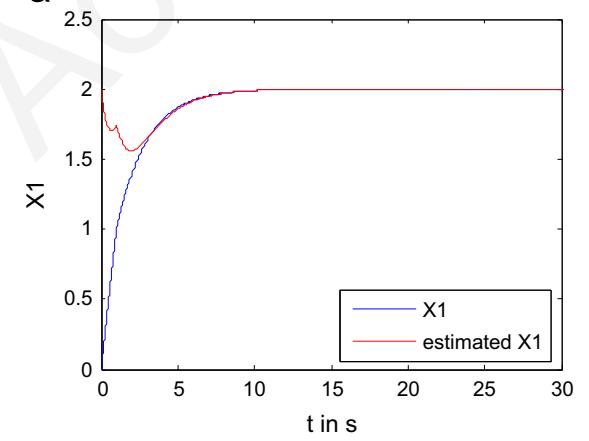

b

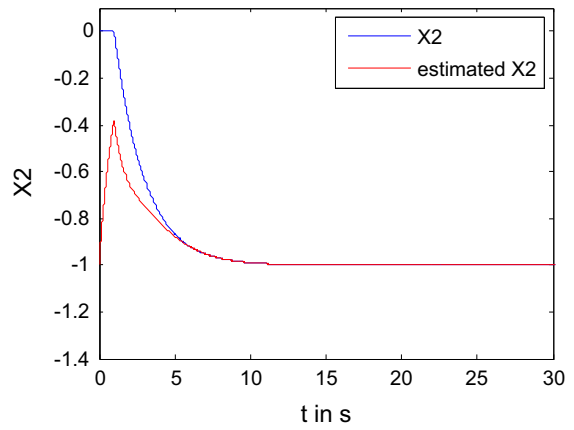

Fig. 1. Example 1: System states and their estimates, observer open loop test. 
a

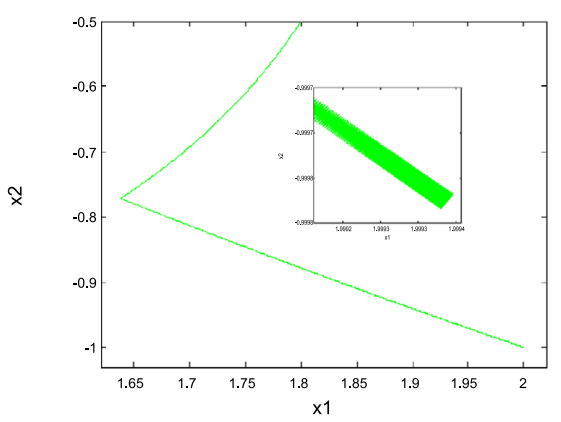

C

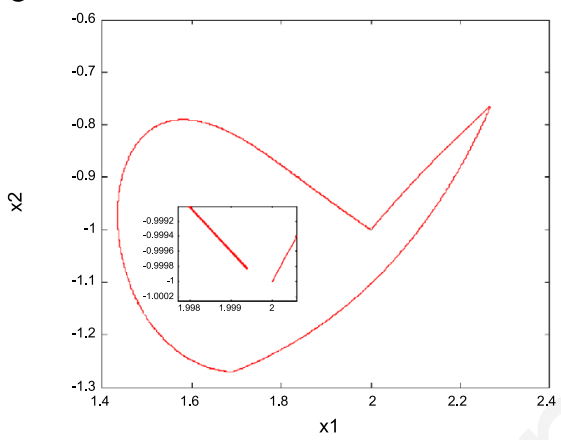

e

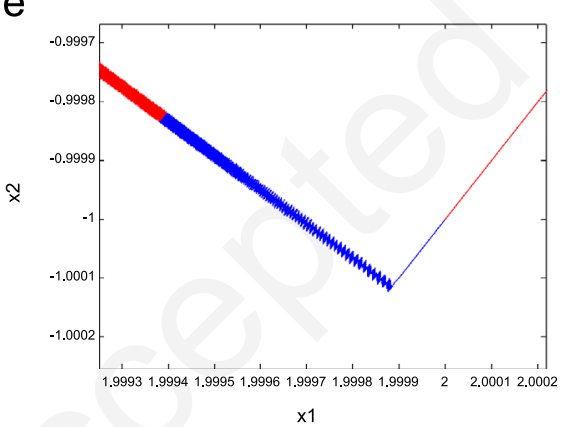

b

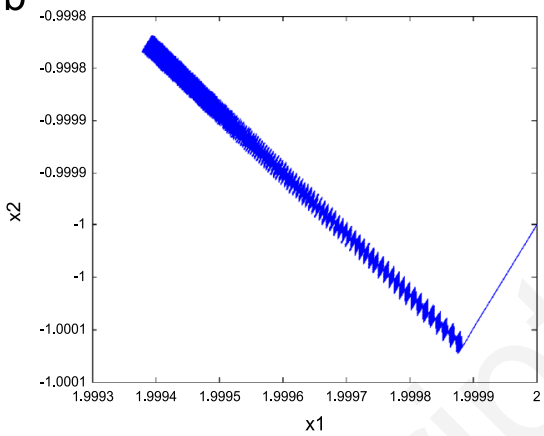

d

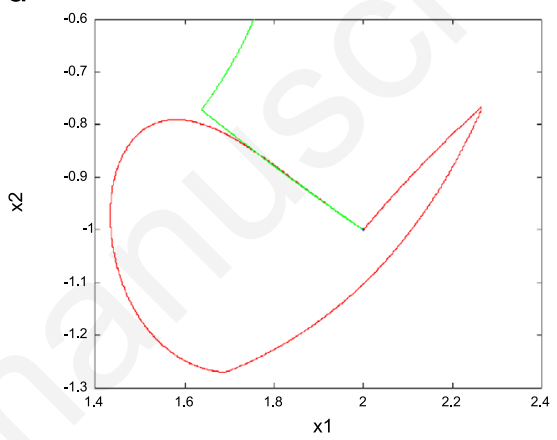

f

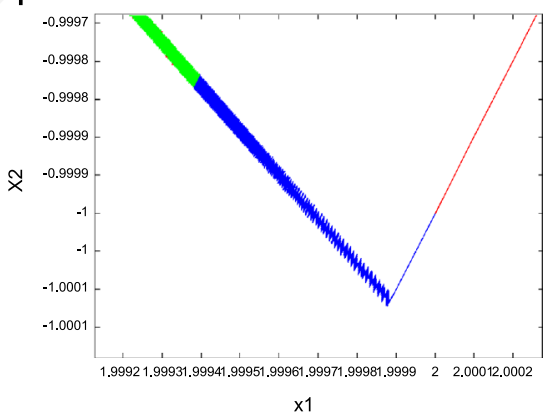

$\times 1$

Fig. 2. Example 1: Trajectories with high precision: (a) stabilization without observer, (b) stabilization based observer with same IC and different ones in (c), Superposition of three executions in (d), Zoom meeting (b) and (c) in (e) and zoom meeting (b) and (c) with (a) in (f).

switching issues [31-33]. Most of these researches are time-domain based on LyapunovKrasovskii Functional Method and Razumikhin theorem, they are generally performed for a specific situation. Fortunately, several authors [34] affirm that for delay-free switched systems, switching strategy may still functions for the delayed version if the time delay is small. Moreover, some delay-dependent dwell time switching strategies to compensate asynchronous switching have also been proposed recently in [35].

On the other parts, showing robustness under invariance principle or robustness of the obtained invariant sets is not an easy task. Some results on the computation of robust invariant 

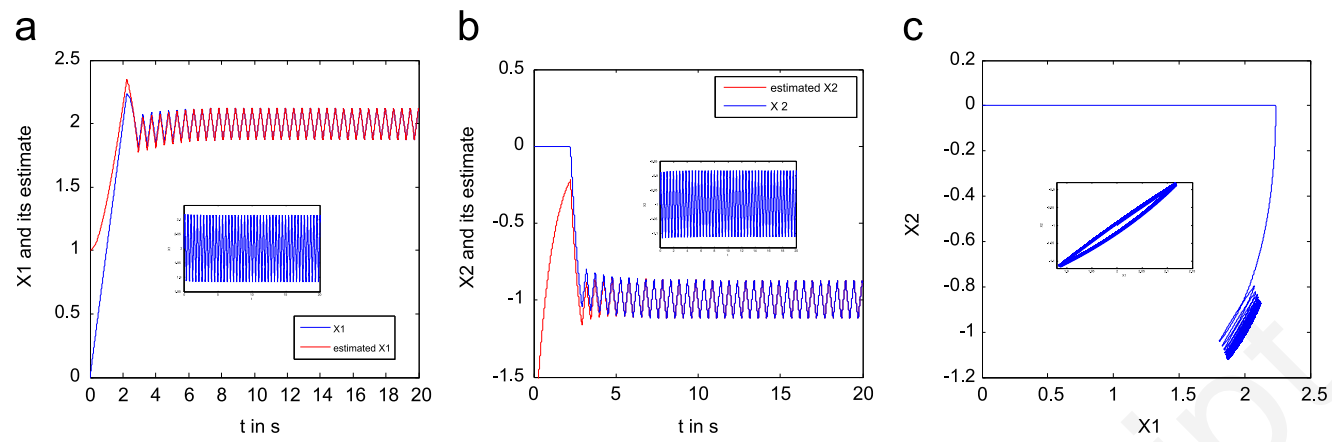

Fig. 3. Example 1: System states and theirs estimates for an execution with less small bound on $\varepsilon_{\mathrm{p}}$, the zoom part for an execution starting near the reference.

sets and/or viability verification that ensures robustness are proposed [36], but still much more works have to be done.

From the above brief discussion, it is clear that addressing all these issues in one unified work is fruitless. Particularly, designing a robust PWA state feedback control for a PWA time-delay system and formulating it as a convex feasibility and/or optimization program is not yet conceivable. In fact, the various promising methods that have emerged for the synthesis of PWA systems are performed for the idealized cases and generally closing with a non-convex problem formulation. An exception is the PWA slab systems subclass, where state feedback quadratic stabilization is formulated as a convex optimization problem [37].

A persistent notice that worth being revealed, is the investigation of the time delay influence on the oscillations occurrence, this may be recognized as a deficient task that may complete this work. while, in the theory of dynamical systems, it is confirmed that varying time delay value can facilitate controlling the system behavior and possibly suppress oscillations, at present, this cannot be confirmed for switched and PWA systems.

\section{Illustrative examples}

Example 1. Consider the following bimodal PWA system:

$$
\left.\dot{\mathrm{x}}(\mathrm{t})=\begin{array}{cc}
0 & \rho \\
-\rho & -1
\end{array}\right) \mathrm{x}+\left(\begin{array}{c}
1-\rho \\
0
\end{array}\right), x \in \mathrm{R}^{2}, \rho \in(0,1)
$$

to be stabilized at an average equilibrium $\mathrm{x}_{\mathrm{e}}=\left(\begin{array}{c}\mathrm{x}_{1} \\ \mathrm{x}_{2}\end{array}\right)=\left(\begin{array}{c}2 \\ -1\end{array}\right)$. For the two approaches, the system has been successfully stabilized from any initial conditions; the Fig. 1 shows the simulation results for the second approach.

According to our idea, the trajectory converges necessary to a small ball around $\mathrm{z}^{* *}$. However, due to the small value of $\varepsilon_{\mathrm{p}}$ we cannot distinguish $\mathrm{z}^{* *}$ from $\mathrm{z}^{*}$, so that it seems that we have a stabilization around the average equilibrium desired reference $z^{*}$. More precise results are showed in Fig. 2. In fact, Fig. 2-a shows stabilization based on system states i.e. without observer. Fig. 2-b represents trajectory when stabilization is based on observer output, the system 


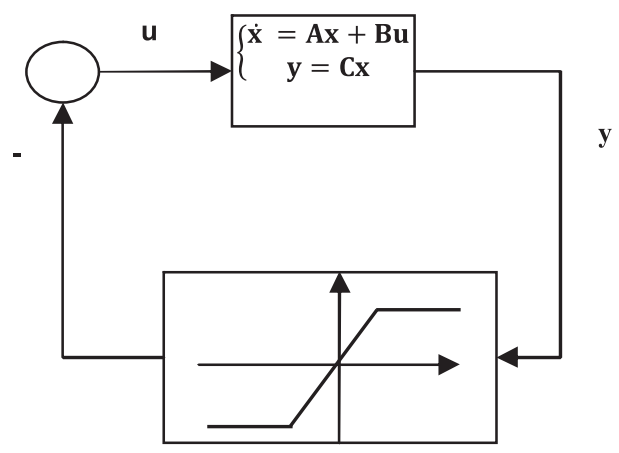

Fig. 4. Example 2: a general feedback interconnection of a saturating system.
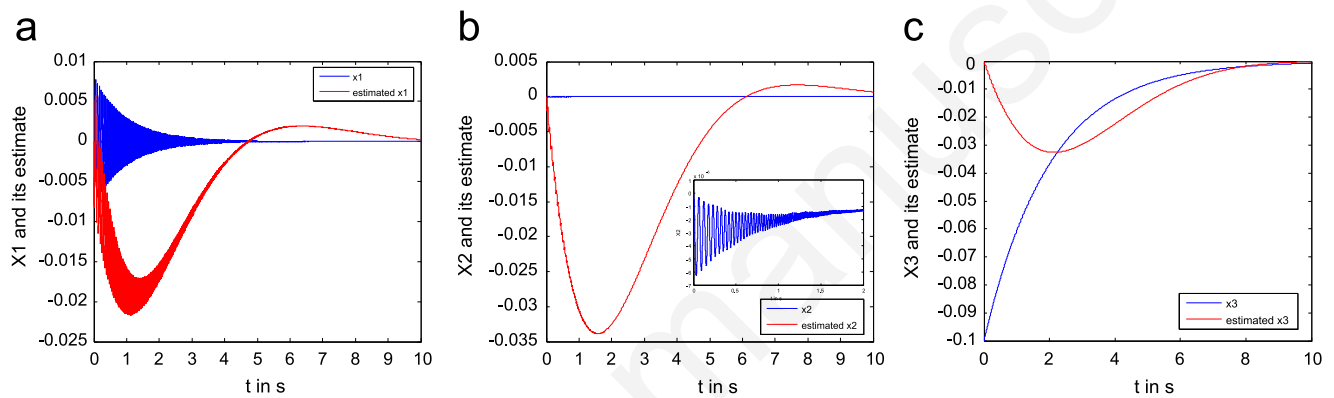

Fig. 5. Example 2: System states and theirs estimate for the stabilization at the origin, observer open loop test.

and its states estimator start from the same initial conditions. Whereas in Fig. 2-c, the stabilization based observer is performed when the system and observer start from different initial conditions. A superposition of the three execution is presented in fig 2-d, where the second execution is scarcely visible.

A confirmation that trajectories of all three executions converge to the same convergence ball around $\mathrm{z}^{* *}$ (propriety of invariance) is illustrated by Fig. 2-e and Fig. 2-f. Where trajectory 2-b meets trajectory 2-c on the convergence ball in Fig. 2-e. The two resultant trajectories meet trajectory 2-a on the same ball in Fig. 2-f.

When the optimization is performed for a less small bound value on $\varepsilon_{\mathrm{p}}$, the switching effects and the periodicity of the trajectory are more visible, see Fig. 3. As well, if starting in the vicinity of the desired average equilibrium and the working precision is very small, the cyclic evolution is provoked and the occurrence of limit cycle is clearly recognizable, see the zoom part in Fig. 3. These oscillations are around the average equilibrium desired reference $z^{*}$ as stated in the theorem 1.

\section{Example 2. Saturating Systems}

Consider a general feedback interconnection of an LTI siso system from [38] with a saturation controller as depicted in Fig. 4. Recall, Symmetric and asymmetric saturation exists in different parts of a system control, such as the actuator, the sensor and the controller. However, most attention has been devoted to symmetric actuator saturation to which may be transformed also 

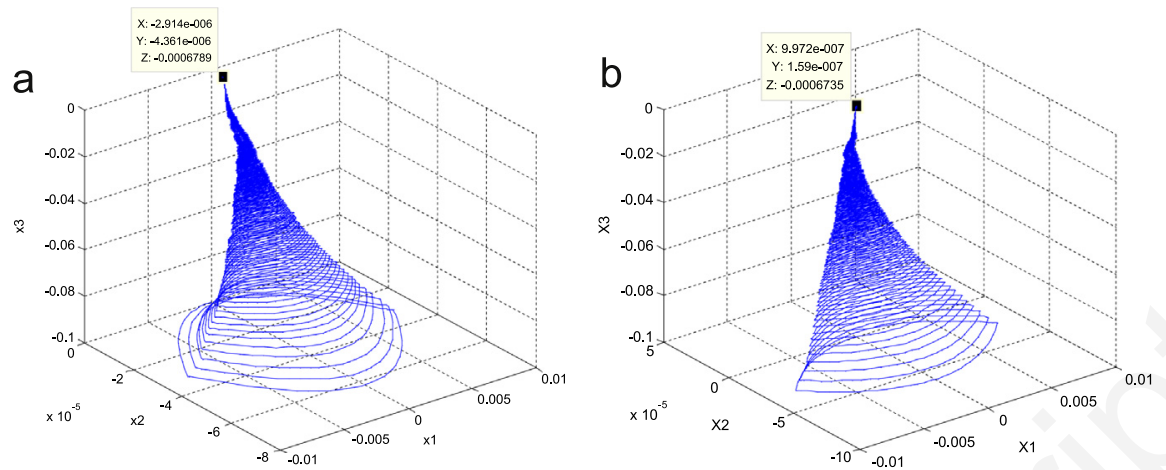

Fig. 6. Example 2: (a) trajectory for stabilization based on system states, (b) trajectory for stabilization based on estimated states.
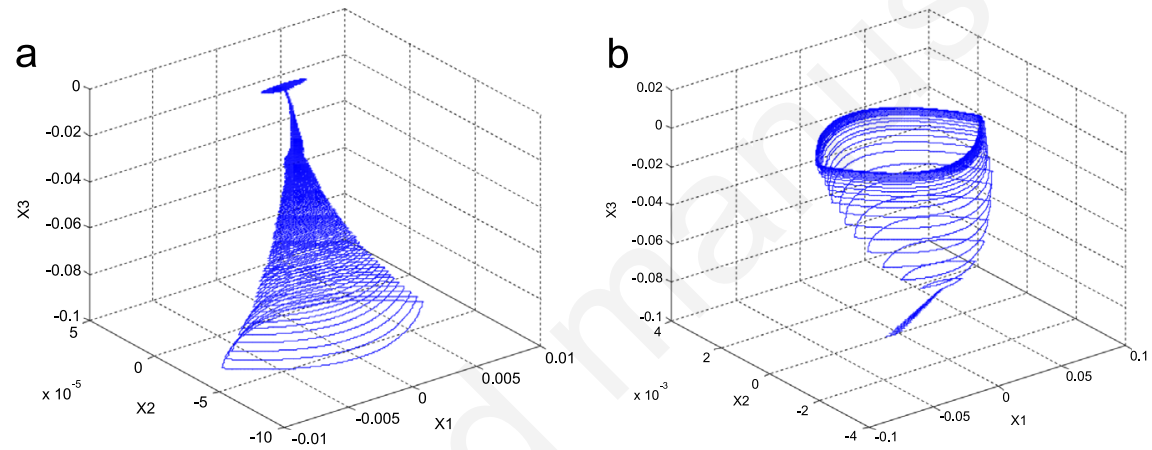

Fig. 7. Example 2: Limit cycles arising for a less precise execution.

the asymmetric one [39]. Actuator saturation involves fundamental control problems such as global stabilization, its effect is often related to physical-controlled systems with input magnitude limitation and networked control systems $[1,40]$.

Where the control signal obey to the following rule for constants $u_{0}, d>0$ :

$$
\mathrm{u}(\mathrm{t})=\left\{\begin{array}{c}
-\mathrm{u}_{0} \text { if } y(\mathrm{t})>d \\
-\frac{\mathrm{u}_{0}}{\mathrm{~d}} \mathrm{y}(\mathrm{t}) \text { if }|\mathrm{y}(\mathrm{t})| \leq \mathrm{d} \\
\mathrm{u}_{0} \text { if } y(\mathrm{t})<-d
\end{array}\right.
$$

We obtain a saturating system which belongs to a strongly nonlinear class of systems; these systems can exhibit extremely complex behaviors. Some may be chaotic, others may have several isolated equilibriums, others might have limit cycles, or even some combination of all these behaviors.

As discussed in [23], this system is symmetric around the origin and has a unique solution for any initial state. In the state space, the saturation controller introduces three regions each with its own dynamic. These regions are separated by two switching surfaces consisting of hyperplanes of dimension $(\mathrm{n}-1): \mathrm{S}_{1.2}=\left\{\mathrm{x} \in \mathrm{R}^{\mathrm{n}} \mid \mathrm{Cx}= \pm \mathrm{d}\right\}$, they can be approximated by conic regions in our 
approach or directly calculated. We obtain the following corresponding PWA system:

$$
\left\{\begin{array} { l } 
{ \dot { \mathrm { x } } = \mathrm { Ax } - \mathrm { Bu } _ { 0 } , \text { for } x \in \Omega _ { 1 } } \\
{ \dot { \mathrm { x } } = ( \mathrm { A } - \frac { \mathrm { u } _ { 0 } } { \mathrm { d } } \mathrm { BC } ) \mathrm { x } , \text { for } x \in \Omega _ { 2 } } \\
{ \dot { \mathrm { x } } = \mathrm { Ax } + \mathrm { Bu } _ { 0 } , \text { for } x \in \Omega _ { 3 } }
\end{array} \text { with } \left\{\begin{array}{c}
\Omega_{1}=\left\{\mathrm{x} \in \mathrm{R}^{\mathrm{n}} \mathrm{Cx}>d\right\} \\
\Omega_{2}=\left\{\mathrm{x} \in \mathrm{R}^{\mathrm{n}}|| \mathrm{Cx} \mid \leq \mathrm{d}\right\} \\
\Omega_{3}=\left\{\mathrm{x} \in \mathrm{R}^{\mathrm{n}} \mathrm{Cx}<d\right\}
\end{array}\right.\right.
$$

A deeper investigation of the system behavior is strongly related to the examination of the eigenvalues of the matrix $\left(A-\frac{u_{0}}{d} B C\right)$. This allows deriving conditions and state space subsets from which the system can converge asymptotically to the origin, to a limit cycle or even diverge see [23] for more detail. Yet, most approaches fall to stabilize this system at the origin which represents an average equilibrium.

\subsection{Numerical application: "third order Goodwin oscillator model"}

This example is taken from [38] as a saturation system with

$$
\mathrm{A}=\left(\begin{array}{ccc}
-0.5 & 0 & 0 \\
0.5 & -0.5 & 0 \\
0 & 0.5 & -0.5
\end{array}\right), \quad \mathrm{u}_{0}=\frac{1}{2} \text { and } d=\frac{1}{9}
$$

So that, we have the following PWA system:

$$
\begin{gathered}
\mathrm{A}_{1}=\left(\begin{array}{ccc}
-0.5 & 0 & 0 \\
0.5 & -0.5 & 0 \\
0 & 0.5 & -0.5
\end{array}\right), \quad \mathrm{b}_{1}=\left(\begin{array}{c}
-1 / 2 \\
0 \\
0
\end{array}\right) ; \\
\mathrm{A}_{2}=\left(\begin{array}{ccc}
-0.5 & 0 & -9 / 2 \\
0.5 & -0.5 & 0 \\
0 & 0.5 & -0.5
\end{array}\right), \quad b_{2}=\left(\begin{array}{c}
0 \\
0 \\
0
\end{array}\right) ; \\
\mathrm{x}=\left(\begin{array}{c}
\mathrm{x} 1 \\
\mathrm{x} 2 \\
\mathrm{x} 3
\end{array}\right), \text { for the origin, we have: } \mathrm{x}_{1}=\mathrm{x}_{2}=\mathrm{x}_{3}=0 . \\
0.5 \\
0
\end{gathered}
$$

Unlike most available approaches, our complete methodology can easily be applied to stabilize this PWA system at the origin $\mathrm{x}_{0}=\left(\begin{array}{l}0 \\ 0 \\ 0\end{array}\right)$. First, Fig. 5 and Fig. 6-a (left) show the impeccable result of the control approach to stabilize the system at the origin. The excellent quality of the switched observer can be evaluated from the rapid convergence of the estimation errors in Fig. 5. Finally, the irrefutable confirmation of the methodology when the stabilization is based on observer outputs is shown by Fig. 6-b (right) that represents a closed loop test of the observer.

As stated before, all these stabilization are toward the convergence ball around $z^{* *}$. However, under some conditions the system can oscillate around the average equilibrium $z^{*}$. In the Fig. 7-a (left), we have showed the occurrence of oscillations. By a diminution of the working precision, 
we can clearly visualize the direct passage to the periodic solution which is illustrated by Fig. 7-b (right).

\section{Conclusion}

In this paper, a novel idea was proposed, to characterize periodic solutions and to determine the critical parameters of the cyclic behaviors of PWA systems, under practical stabilizing switching law. It has been demonstrated that the convergence domains of the general approach are invariants and strongly related to the appearance of periodic solutions. Moreover, the proposed approach has an advantage to deal with prescribed oscillation parameters values which can be included directly in the design operation. The different theorems and propositions proposed in this paper have been illustrated by various simulations. Effects of system uncertainties and the influence of time delay during the switching are not considered in this paper. Their impact on the invariant set robustness and the cyclic behaviors of limit points will be a natural extension of this work.

\section{References}

[1] M. Johansson, Piecewise linear control systems: a computational approach, in: M. Thoma, M. Morari (Eds.), Lecture Notes in Control and Information Sciences, Springer-Verlag, 2003 ser.

[2] A. Bemporad, G. Ferrari-Trecate, M. Morari, Observability and controllability of piecewise affine and hybrid systems, IEEE Trans. Autom. Control 45 (2000) 1864-1876.

[3] P. Bolzern, W. Spinelli, Quadratic stabilization of a switched affine system about a nonequilibrium point, in: Proceedings of the American control conference, 2004, pp. 3890-3895.

[4] R.DeCarlo, M.Branicky, S.Pettersson, B.Lennartson, Perspectives and results on the stability and stabilizability of hybrid systems, in: Proceedings of the IEEE. 88 (7), 2000, pp. 1069-1082.

[5] A.Hassibi, S.Boyd, Quadratic stabilization and control of Piecewise-Linear systems, in: Proceedings of the American Control Conference, 1998, pp. 3659-3664.

[6] L. Rodrigues, H.P. Jonathan, Observer-based control of piecewise affine systems, Int. J. Control 76 (2003) $459-477$.

[7] H. Lin, P.J. Antsaklis, stability and stabilizability of switched linear systems, a survey of recent results, IEEE Trans. Autom. Control 54 (2) (2009) 308-322.

[8] R. Goebel, R.G. Sanfelice, A.R. Teel, Hybrid dynamical systems, IEEE Control Syst. Mag. 29 (2) (2009) $28-93$.

[9] H. Fang, Z. Lin, Global practical stabilization of planar linear systems in the presence of actuator saturation and input additive disturbance, Inst. Electr. Electron. Eng. Trans. Autom. Control 51 (7) (2006) 1177-1184.

[10] D. Kamri, R. Bourdais, J. Buisson, C. Larbes, Piecewise-Affine systems practical stabilization: a BMI approach, Nonlinear Anal.: Hybrid. Syst. 6 (2012) 859-870.

[11] D. Kamri, C. Larbes, Observer based control for DC/DC converters: practical switching control, Arab. J. Sci. Eng. 39 (2014) 4089-4102.

[12] G. Zhai, A.N. Michel, Generalized practical stability analysis of discontinuous dynamical systems, Int. J. Appl. Math. Comput. Sci. 1 (14) (2004) 5-12.

[13] X. Xu, G. Zhai, S. He, On practical asymptotic stabilizability of switched affine systems, Nonlinear Anal.: Hybrid. Syst. (2008) 196-208.

[14] R. Kuiava, R.A. Ramos, H.R. Pota, L.F.C. Alberto, Practical stability of continuous-time switched systems without a common equilibria and governed by a time-dependent switching signal, in: Proceedings of the 9th IEEE International Conference on Control and Automation, Santiago, Chilie, 2011.

[15] D. Patino, P. Riedinger, C. Iung, Practical optimal state feedback control law for continuous-time switched affine systems with cyclic steady state, Int. J. Control 7 (82) (2009) 1357-1376.

[16] F. Blanchini, Set invariance in control, Automatica 35 (11) (1999) 1747-1767.

[17] Luis Rodrigues, Stability analysis of piecewise-affine systems using controlled invariant sets, Syst. Control Lett. 53 (2) (2004) 157-169.

[18] S. Sankaranarayanan, Automatic Invariant Generation for Hybrid Systems using Ideal Fixed Points (HSCC'10), ACM221-230. 
[19] L. Berardi, E. De Santis, M.D. Di Benedetto, in: Hybrid Systems with Safety Specifications: Procedures for the Computation and Approximations of Controlled Invariant Sets, Univ. L'Aquila, Dept. Elect. Eng., 2003.

[20] A. Nikos, K. James, V. D. Jyotirmoy, P. André, K. Bruce, Forward invariant cuts to simplify proofs of safety, in: Conference on Embedded Software, EMSOFT '15', IEEE Press, 2015, pp. 227-236.

[21] M.Jirstrand, Invariant Sets for a Class of Hybrid Systems, Report no: LITH_ISY_R2025, March 98 available at: 〈http://www.diva-portal.org/smash/get/diva2:316433/FULLTEXT02〉.

[22] Jinglai Shen, Positive invariance of constrained Affine Dynamics and its applications to hybrid systems and safety verification, IEEE Trans. Autom. Control 57 (1) (2012) 3-18.

[23] M.J. Gonçalves, A. Megretski, M. Dahleh, Global analysis of piecewise linear systems using impact maps and quadratic surface lyapunov functions, IEEE Trans. Autom. Control 12 (48) (2003) 2089-2106.

[24] U.T. Jonsson, A. Megretski, A small-gain theory for limit cycles of systems on lur'e form, SIAM J. Control Optim. 3 (44) (2005) 909-938.

[25] M. Egerstedt, Y. Wardi, H. Axelsson, Transition-time optimization for switched-mode dynamical systems, IEEE Trans. Autom. Control 1 (51) (2006) 110-115.

[26] X. Xu, P.J. Antsaklis, Optimal control of switched systems based on parameterization of the switching instants, IEEE Trans. Autom. Control 1 (49) (2004) 2-16.

[27] J.P. Richard, Time-delay systems: on overview of some recent advances and open problems, Automatica 39 (10) (2003) 1667-1694.

[28] L.V. Hien, Q.P. Ha, V.N. Phat, Stability and stabilization of switched linear dynamic systems with time delay and uncertainties, Appl. Math. Comput. 210 (2009) 223-231.

[29] L.V. Hien, V.N. Phat, Exponential stabilization for a class of hybrid systems with mixed delays in state and control, Nonlinear Anal.: Hybrid. Syst. 3 (2009) 259-265.

[30] K. Moezzi, L. Rodrigues, A.G. Aghdam, Stability of uncertain piecewise affine systems with time-delay: delaydependent Lyapunov approach, Int. J. Control 82 (8) (2009) 1423-1434.

[31] L.X. Zhang, H.J. Gao, Asynchronously switched control of switched linear systems with average dwell time, Automatica 46 (5) (2010) 953-958.

[32] L. Vu, M.A. Kristi, Stability of time-delay feedback switched linear systems, IEEE Trans. Autom. Control 55 (10) (2010) 2385-2389.

[33] Y.E.Wang, X.M.Sun and J.Zhao, H. Asynchronous, E.Wang, X.M.Sun and J.Zhao, Asynchronous feedback switched linear systems, IEEE Transactions on Automatic C69.

[34] S. Kim, S.A. Campbell, X. Liu, Stability of a class of linear switching systems with time-delay, IEEE Trans. Circuits Syst.-I 53 (2) (2006) 384-393.

[35] Li-Li Liu, Yue-E. Wang, Bao-Wei Wu, Asynchronous stabilization of switched singular systems with interval timevarying delays, Int. J. Control Autom. 8 (11) (2015) 373-384.

[36] T.Alamo, A.Cepeda, D.Limon, J.M.Bravo, M.Fiacchini, E.F.Camacho, On the computation of robust control invariant sets for piecewise affine systems, Workshop on Assessment and Future Directions on NMPC, FreudentadtLauterbad, Germany, 2005, pp. 26-30.

[37] B. Samadi, L.Rodrigues, Controller synthesis for piecewise affine slab differential inclusions: a duality-based convex optimization approach, in: Proceedings of the 46th IEEE Conference on Decision and Control, IEEE, 2007, pp. 4999-5004.

[38] A.A. Salinas-Varela, G.B. Stan, M.J. Gonçalves, Global Asymptotic Stability of the Limit Cycle in Piecewise Linear versions of the Goodwin Oscillator, Proceedings of the 17th IFAC World Congress, Seoul, Korea, 3659-3664.

[39] Y. Chengzhi, W. Fen, Switching control of linear systems subject to asymmetric actuator saturation, Int. J. Control 88 (1) (2015) 204-215.

[40] S. Tarbouriech, G. Garcia, A.H. Glattfelder, Advanced Strategies in Control Systems with Input and Output Constraints, vol. 346 of Lecture Notes in Control and Information Sciences, Springer, Berlin, Germany, 2007. 